\title{
Generalizations of Wiener polarity index and terminal Wiener index
}

\author{
Aleksandar Ilić $\ddagger$ \\ Faculty of Sciences and Mathematics, Višegradska 33, 18000 Niš \\ University of Niš, Serbia \\ e-mail: aleksandari@gmail.com \\ Milovan Ilić \\ Faculty of Information Technology, Trg republike 3, 11000 Beograd \\ University of Belgrade, Serbia \\ e-mail: ilic.milovan@gmail.com
}

October 17, 2018

\begin{abstract}
In theoretical chemistry, distance-based molecular structure descriptors are used for modeling physical, pharmacologic, biological and other properties of chemical compounds. We introduce a generalized Wiener polarity index $W_{k}(G)$ as the number of unordered pairs of vertices $\{u, v\}$ of $G$ such that the shortest distance $d(u, v)$ between $u$ and $v$ is $k$ (this is actually the $k$-th coefficient in the Wiener polynomial). For $k=3$, we get standard Wiener polarity index. Furthermore, we generalize the terminal Wiener index $T W_{k}(G)$ as the sum of distances between all pairs of vertices of degree $k$. For $k=1$, we get standard terminal Wiener index. In this paper we describe a linear time algorithm for computing these indices for trees and partial cubes, and characterize extremal trees maximizing the generalized Wiener polarity index and generalized terminal Wiener index among all trees of given order $n$.
\end{abstract}

Key words: Distance in graphs; Wiener polarity index; terminal Wiener index; Wiener index; Partial cube; Graph algorithm.

AMS Classifications: 05C12, 92E10.

\section{Introduction}

Let $G=(V, E)$ be a connected simple graph with $n=|V|$ vertices and $m=|E|$ edges. For vertices $u, v \in V$, the distance $d(u, v)$ is defined as the length of the shortest path between $u$ and $v$ in $G$. The diameter $\operatorname{diam}(G)$ is the greatest distance between two vertices of $G$. Let $d_{k}(u)$ denotes the number of vertices on distance $k$ from the vertex $u$. Let $\operatorname{deg}(v)$ denotes the degree of the vertex $v$.

In theoretical chemistry molecular structure descriptors (also called topological indices) are used for modeling physico-chemical, pharmacologic, toxicologic, biological and other properties of chemical compounds [11. There exist several types of such indices, especially those based on vertex and edge distances. Arguably the best known of these indices is the Wiener index $W$, defined as the sum of distances between all pairs of vertices of the molecular 
graph 9 ]

$$
W(G)=\sum_{u, v \in V(G)} d(u, v) .
$$

Besides of use in chemistry, it was independently studied due to its relevance in social science, architecture and graph theory. With considerable success in chemical graph theory, various extensions and generalizations of the Wiener index are recently put forward [3, 24].

The Wiener polarity index of a graph $G$ is defined as the number of unordered pairs of vertices $\{u, v\}$ of $G$ such that the shortest distance $d(u, v)$ between $u$ and $v$ is 3 ,

$$
W P(G)=|\{(u, v) \mid d(u, v)=3, u, v \in V\}| .
$$

Hosoya [12] found a physico-chemical interpretation of $W P$. Du, Li and Shi [8] described a linear time algorithm for computing the Wiener polarity index of trees, and characterized the trees maximizing the index among all trees of given order. Deng et al. [4, 5, 6] and Liu et al. 20] characterized extremal $n$-vertex trees with given diameter, number of pendent vertices or maximum vertex degree.

For $k \geq 1$, we define the generalized Wiener polarity index as the number of unordered pairs of vertices $\{u, v\}$ of $G$ such that the shortest distance $d(u, v)$ between $u$ and $v$ is $k$,

$$
W_{k}(G)=\frac{1}{2} \sum_{v \in V(G)} d_{k}(v)=|\{(u, v) \mid d(u, v)=k, u, v \in V\}| .
$$

Notice that $W(G)=\sum_{k=1}^{\operatorname{diam}(G)} W_{k}(G)$. If $x$ is a parameter, then the Wiener polynomial of $G$ is defined as [13, 22]

$$
W(G, x)=\sum_{u, v \in V(G)} x^{d(u, v)}=\sum_{k=1}^{\operatorname{diam}(G)} W_{k}(G) \cdot x^{k} .
$$

Therefore, the generalized Wiener polarity index is basically the $k$-th coefficient in the Wiener polynomial.

The terminal Wiener index of a graph $G$ is defined by Gutman, Furtula and Petrovic in [10] as the sum of distances between all pairs of pendent vertices of $G$,

$$
T W(G)=\sum_{\begin{array}{c}
u, v \in V(G) \\
\operatorname{deg}(u)=\operatorname{deg}(v)=1
\end{array}} d(u, v) .
$$

Furthermore, the authors described a simple method for computing TW of trees and characterized the trees with minimum and maximum $T W$. Recently Deng and Zhang [7] studied equiseparability on terminal Wiener index. Independently, Székely et al. [23] introduced the same index (the sum of distances between the leaves of a tree) and studied the correlation between various distance-based topological indices.

For $k \geq 1$, we define the generalized terminal Wiener index as the sum of the distances between all unordered pairs of vertices of degrees $k$,

$$
T W_{k}(G)=\sum_{\begin{array}{c}
u, v \in V(G) \\
\operatorname{deg}(u)=\operatorname{deg}(v)=k
\end{array}} d(u, v) .
$$

The paper is organized as follows. In Section 2 we introduce generalization of the Wiener polarity index $W_{k}(G)$ and characterize the trees maximizing the generalized Wiener polarity 
index among all trees of given order, while in Section 3 we designed linear algorithm for calculating this index. In Section 4 we introduce generalization of the terminal Wiener index and characterize trees maximizing the generalized terminal Wiener index among all trees of given order. In Section 5 we present formula for calculation of $T W_{k}(G)$ for partial cubes and in particular closed formula for $T W_{3}$ of coronene series $H_{k}$. We close the paper in Section 6 by proposing new problems for research.

\section{Generalization of Wiener polarity index}

For $k=1$, it can be easily seen that $W_{1}(G)=m$, where $m$ is the number of edges. For $k=2$, we have

$$
W_{2}(G)=\sum_{v \in V}\left(\begin{array}{c}
\operatorname{deg}(v) \\
2
\end{array}\right)=\frac{\sum_{v \in V} \operatorname{deg}^{2}(v)}{2}-m=\frac{M_{1}(G)}{2}-m,
$$

where $M_{1}(G)$ denotes the first Zagreb index of a graph [21].

For $k=3$ we have the Wiener polarity index,

$$
\begin{aligned}
W_{3}(T) & =\sum_{u v \in E}(\operatorname{deg}(v)-1)(\operatorname{deg}(u)-1)=\sum_{u v \in E} \operatorname{deg}(u) \operatorname{deg}(v)-\sum_{v \in V} \operatorname{deg}^{2}(v)+m \\
& =M_{2}(T)-M_{1}(T)+m,
\end{aligned}
$$

where $M_{2}(T)$ denotes the second Zagreb index of a graph [14].

In the following assume that $k \geq 3$. If the diameter of $T$ is less than $k$, then $W_{k}(T)=0$. Therefore, the minimum value of $W_{k}(T)$ is zero, and it is achieved for all trees with $\operatorname{diam}(T)<$ $k$ (for example the star $S_{n}$ ). On the other hand, we will prove that the maximum value of $W_{k}(T)$ is achieved for a tree with diameter $k$ and with all pendent vertices on distance $k$.

The group of pendent vertices is defined as the set of all pendent vertices attached to the same unique neighbor. Let $A_{1}$ and $A_{2}$ be two different groups of pendent vertices with the unique neighbors $w_{1}$ and $w_{2}$, such that the distance between two arbitrary pendent vertices from these groups is not equal to $k$. Let $p_{1}$ be the number of vertices on distance $k$ from an arbitrary pendent vertex from $A_{1}$ and $p_{2}$ be the number of vertices on distance $k$ from an arbitrary pendent vertex from $A_{2}$. Without loss of generality assume that $p_{1} \leq p_{2}$. If we remove all pendent vertices from $A_{2}$ and add them to the group $A_{1}$, we get a new tree $T^{\prime}$ such that

$$
W_{k}\left(T^{\prime}\right)-W_{k}(T)=\left(\left|A_{1}\right| p_{1}+\left|A_{2}\right| p_{2}\right)-\left(\left|A_{1}\right| p_{1}+\left|A_{2}\right| p_{1}\right)=\left|A_{2}\right|\left(p_{2}-p_{1}\right) \geq 0 .
$$

By repetitive application of this transformation, we will get a new tree with possibly increased generalized Wiener polarity index. The diameter of $T^{\prime}$ is not greater than the diameter of $T$ and each transformation introduces one new pendent vertex. By choosing two most distant groups of pendent vertices, we will get the extremal tree with diameter equal to $k$. After that we can apply the transformation finitely many times, until all pendent vertices are on distance $k$ or 2 .

Assume that there are $p$ groups of pendent vertices with sizes $a_{1}, a_{2}, \ldots, a_{p}$ and $a_{1}+a_{2}+$ $\ldots+a_{p}=q$. Since $\operatorname{diam}(T)=k$, we have $n-k+1 \geq q \geq 2$. The distance between any two pendent vertices not from the same group is equal to $k$, and therefore

$$
W_{k}(T)=\frac{1}{2} \sum_{i=1}^{p} a_{i}\left(q-a_{i}\right)=\frac{1}{2}\left(q^{2}-\sum_{i=1}^{p} a_{i}^{2}\right) .
$$


The minimum value of $\sum_{i=1}^{p} a_{i}^{2}$ under the condition $\sum_{i=1}^{p} a_{i}=q$ is achieved if and only if all numbers $a_{i}$ are as close as possible, i. e. $\left|a_{i}-a_{j}\right| \leq 1$ for all $1 \leq i \leq j \leq p$. This can be easily proved by the transformation $\left(a_{i}, a_{j}\right) \mapsto\left(a_{i}+1, a_{j}-1\right)$ with $a_{j} \geq a_{i}+2$, since

$$
\left(a_{i}+1\right)^{2}+\left(a_{j}-1\right)^{2}-a_{i}^{2}-a_{j}^{2}=2\left(a_{i}-a_{j}\right)+2<0 .
$$

Notice that the tree is uniquely determined by the distances between pendent vertices [26]. A starlike tree is a tree with exactly one vertex of degree $\geq 3$. If $p=2$, we have $W_{k}(T)=a_{1} a_{2}$ and $a_{1}+a_{2}=n-k+1$ and finally

$$
W_{k}(T)=\left\lfloor\frac{n-k+1}{2}\right\rfloor \cdot\left\lceil\frac{n-k+1}{2}\right\rceil
$$

Let $p>2$. For $k$ odd, we can consider two groups of pendent vertices together with the unique path connecting them. The third group of pendent vertices must be on equal distance from both groups and that is impossible. Therefore, the extremal value for odd $k$ is achieved for $p=2$.

For $k$ even, similarly it can be concluded that there is a unique tree with $p$ groups of pendent vertices (starlike tree with $p$ paths with equal lengths together with groups of pendent vertices attached at the end vertices of these $p$ paths). For $p>2$, we have

$$
n=1+p\left(\frac{k}{2}-1\right)+\sum_{i=1}^{p} a_{i}=1+p\left(\frac{k}{2}-1\right)+q,
$$

and since $p \leq q$, we have $p \leq 2 \cdot \frac{n-1}{k}$. Therefore, using Cauchy-Schwartz inequality it follows

$$
\begin{aligned}
W_{k}(T) & =\frac{1}{2}\left(q^{2}-\sum_{i=1}^{p} a_{i}^{2}\right) \\
& \leq \frac{1}{2}\left(q^{2}-\frac{q^{2}}{p}\right) \\
& =\frac{1}{2}\left(n-1-\frac{p k}{2}+p\right)^{2}\left(1-\frac{1}{p}\right) .
\end{aligned}
$$

Let

$$
f(p)=\frac{1}{2}\left(n-1-\frac{p k}{2}+p\right)^{2}\left(1-\frac{1}{p}\right)
$$

for $2<p<2 \cdot \frac{n-1}{k}<2 \cdot \frac{n-1}{k-2}$. The first derivative equals

$$
f^{\prime}(p)=\frac{(2-2 n-2 p+k p)\left(2-2 n+2 p-k p-4 p^{2}+2 k p^{2}\right)}{8 p^{2}} .
$$

Finally it holds that $f(p)$ is increasing for $2<p<p^{*}$ and decreasing for $p^{*} \leq p<2 \cdot \frac{n-1}{k}$, where

$$
p^{*}=\frac{k-2+\sqrt{(k-2)(16 n+k-18)}}{4(k-2)}=\frac{1}{4}+\frac{1}{4} \sqrt{\frac{16 n+k-18}{k-2}}
$$

is the second largest root of $f^{\prime}(p)=0$. Therefore, the maximum of the Wiener polarity index for $k$ even should be achieved for $p=2$ or integers around $p^{*}$ (see Figure 1). 


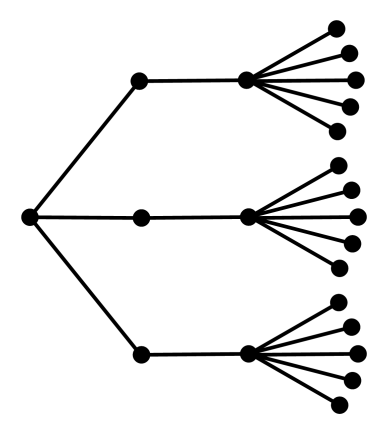

Figure 1: The extremal tree with maximal generalized Wiener polarity index on $n=22$ and $k=6$.

\section{Linear algorithm for $W_{k}$ of trees}

Let $T$ be an arbitrary tree rooted at the vertex 1 . We will process the vertices according to the distance from the root vertex or in the recursive depth first search method [2]. For each vertex $v$, keep the vector $a[v]$ of the length $k+1$ that stores the number of vertices in the subtree under $v$ on distances $0,1,2, \ldots, k$. It follows that $a[v][0]=1$ and $a[v][1]=\operatorname{deg}(v)-1$ for all vertices different than the root. The matrix $a$ with dimensions $n \times k$ is computed recursively in the first procedure.

In the second procedure we calculate the generalized Wiener polarity index. In DFS tree, for each path of the length $k$ there is a unique vertex $v$ on the smallest distance from the root. Therefore, we need to traverse all vertices $v$ and count the vertices that are on distance $k$ in the subtree under $v$, such that the unique path connecting these vertices contains $v$. For the vertices in the subtree under $v$, we just add $2 a[v][k]$. Otherwise, we need to consider all neighbors $u$ of $v$ different than parent $[v]$ and for each $i=0,1, \ldots, k-2$ count the number of vertex pairs $(x, y)$ such that:

- $x$ is in the subtree under $u$ and $y$ is not;

- $x$ is on the distance $i$ from $u$;

- $y$ is on the distance $k-i-1$ from $v$ and under $v$.

Finally we counted every vertex pair on distance $k$ twice, as showed in the second procedure. The time and memory complexity is $O(n k)$.

\section{Generalization of terminal Wiener index}

For $k$ regular graphs, $T W_{k}(G)=W(G)$ and $T W_{i}(G)=0$ for $i \neq k$. For $k=1$, we have terminal Wiener index.

Theorem 4.1 ([9]) Let $T$ be a tree on $n$ vertices. Then,

$$
(n-1)^{2}=W\left(S_{n}\right) \leq W(T) \leq W\left(P_{n}\right)=\left(\begin{array}{c}
n+1 \\
3
\end{array}\right),
$$

with equality if and only if $T \cong S_{n}$ or $T \cong P_{n}$.

Theorem 4.2 ([15]) Let $w$ be a vertex of a nontrivial connected graph $G$. For nonnegative integers $p$ and $q$, let $G(p, q)$ denote the graph obtained from $G$ by attaching to vertex $w$ pendent 

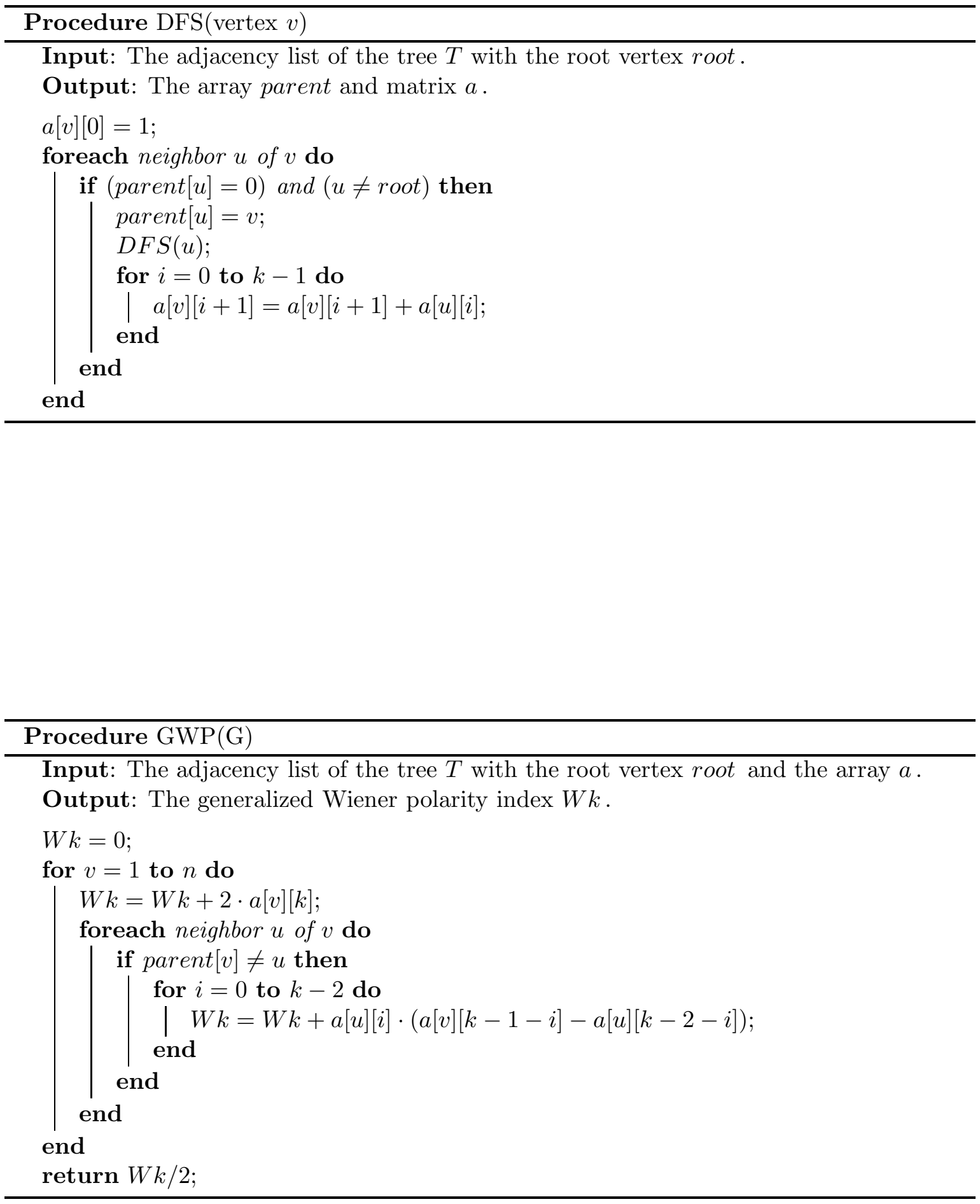
paths $P=w v_{1} v_{2} \ldots v_{p}$ and $Q=w u_{1} u_{2} \ldots u_{q}$ of lengths $p$ and $q$, respectively. If $p \geq q \geq 1$, then

$$
W(G(p, q))<W(G(p+1, q-1)) .
$$

For $k=1$, Gutman, Furtula and Petrović in [10] characterized the extremal trees within the class of all trees with $n$ vertices that maximize and minimize terminal Wiener index. The path $P_{n}$ is the unique tree that minimizes $T W_{1}$.

For $k=2$, the unique tree that maximizes $T W_{2}$ is the path $P_{n}$. This follows from Theorems 4.1 and the simple fact that in every tree there are at least 2 pendent vertices (there are at most $n-2$ vertices of degree two, and the maximum sum between all such pairs is achieved for path).

Let $T^{*}$ be the extremal tree that maximizes the generalized terminal Wiener index for $k \geq 3$. It can be easily proved that there are no pendent paths $P=v_{1} v_{2} \ldots v_{p}$ of length greater than 2 or equal to attached at some vertex of $T^{*}$. Otherwise, remove pendent edges one by one and subdivide any edge $e$ such that both components of $T^{*}-e$ contain vertices of degree $k$ - this way we increase $T W_{k}$. If such edge does not exist, remove the edge $v_{p-1} v_{p}$ and add new edge $v_{p-2} v_{p}$ - this way the generalized terminal Wiener index remains the same, or increases if and only if $k=3$.

Lemma 4.3 Among trees on $n$ vertices the maximal possible number of vertices of degree $k \geq 2$ is

$$
m(n, k)=\left\lfloor\frac{n-2}{k-1}\right\rfloor .
$$

Proof. Consider the induced graph $H$ composed of the vertices of degree $k$. Let $h$ be the number of vertices in $H$, and let $f$ be the number of edges in $H$. Since $H$ is acyclic and possible disconnected, we have $0 \leq f \leq h-1$. Furthermore, for the number of edges in tree $T$ holds

$$
h \cdot k-(h-1) \leq h \cdot k-f \leq n-1 .
$$

It follows that $h(k-1) \leq n-2$, which completes the proof.

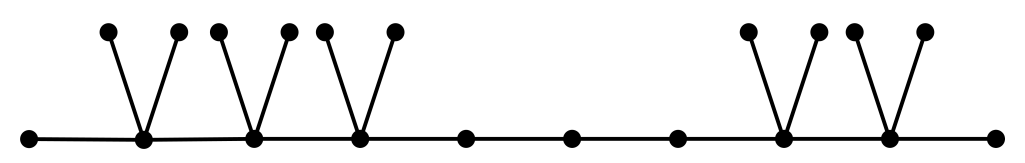

Figure 2: $\quad$ The extremal tree with maximal generalized terminal Wiener index with $n=20$ and $k=4(s=8$ and $p=5)$

Let $C_{n, k, p}$ be the caterpillar obtained from a path of length $s+2=n-p \cdot(k-2)$, by attaching $k-2$ pendent vertices to exactly $p$ vertices of a path $P_{s+2}=v_{0} v_{1} \ldots v_{s} v_{s+1}$, starting from the vertices $v_{1}$ and $v_{s}$ on the both ends towards center symmetrically (see Figure 2). The generalized terminal Wiener index can be easily calculated by considering the pairs of vertices $\left(v_{1}, v_{s}\right),\left(v_{2}, v_{s-1}\right), \ldots$ and summing the distance between all intermediate vertices: 


$$
\begin{aligned}
T W_{k}\left(C_{n, k, p}\right) & =\sum_{1 \leq i, j, \leq s} d\left(v_{i}, v_{j}\right) \\
& =(p-1)(s-1)+(p-3)(s-3)+(p-5)(s-5)+\ldots \\
& = \begin{cases}\frac{1}{12} p\left(3 p s-p^{2}-2\right), & \text { if } p \text { is even } \\
\frac{1}{12}(p+1)(p-1)(3 s-p), & \text { if } p \text { is odd. }\end{cases} \\
& = \begin{cases}\frac{1}{12} p\left(3 n p+5 p^{2}-3 k p^{2}-2-6 p\right), & \text { if } p \text { is even } \\
\frac{1}{12}(p+1)(p-1)(3 n+5 p-3 k p-6), & \text { if } p \text { is odd. }\end{cases}
\end{aligned}
$$

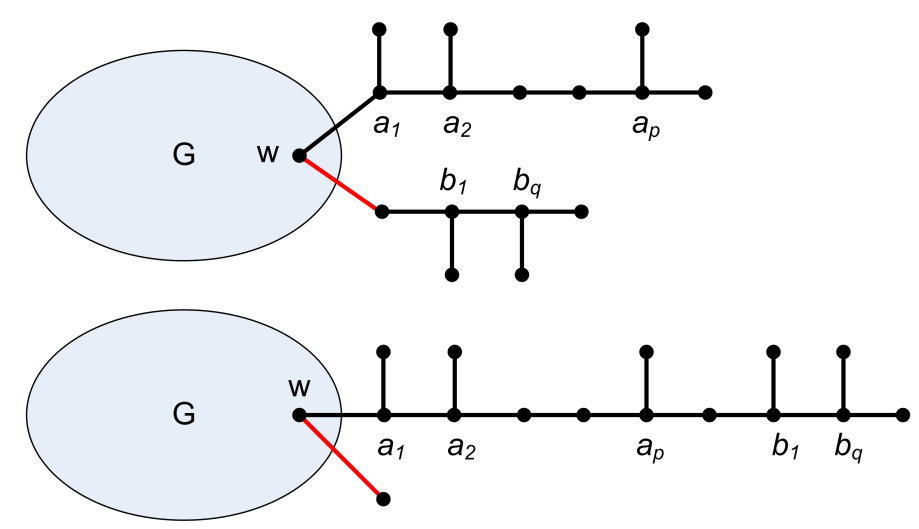

Figure 3: Transformation that increases generalized terminal Wiener index $\mathrm{TW}_{3}$.

We call a caterpillar $C$ 3-bounded if all vertices of $C$ have degree less than or equal to 3 .

Theorem 4.4 Let $T$ be a tree on $n>4$ vertices. Then

$$
T W_{3}(T) \leq T W_{3}\left(C_{n, 3,\lfloor n / 2\rfloor-1}\right),
$$

with equality if and only if $T \cong C_{n, 3,\lfloor n / 2\rfloor-1}$.

Proof. Let $T^{*}$ be a rooted tree with maximal value of generalized terminal Wiener index for $k=3$. We can also assume that the number of vertices of degree 3 is greater than two.

If $T^{*}$ is not 3-bounded caterpillar, consider a branching vertex $w$ such that in the subtree under $w$ there are only 3-bounded caterpillars attached at $w$ (it can happen that there are only pendent vertices attached to $w$ ). Let $C_{1}$ and $C_{2}$ be two caterpillars attached at $w$, such that $C_{1}$ has $p$ vertices $v_{1}, v_{2}, \ldots, v_{p}$ of degree 3 and $C_{2}$ has $q$ vertices $u_{1}, u_{2}, \ldots, u_{q}$ of degree 3 (see Figure 3). Without loss of generality, we can assume that the number of vertices of degree 3 in $C_{1}$ is greater than or equal to the number of vertices of degree 3 , namely $p \geq q$.

Let $a_{1}, a_{2}, \ldots, a_{p}$ be the distances from the vertex $w$ to the vertices $v_{1}, v_{2}, \ldots, v_{p}$ and $b_{1}, b_{2}, \ldots, b_{q}$ be the distance from the vertex $w$ to the vertices $u_{1}, u_{2}, \ldots, u_{q}$. Let $D(w)$ be the sum of distances from the vertex $w$ to the vertices of degree 3 in the subgraph $G$ (see Figure 3).

The generalized terminal Wiener index of the tree $T^{*}$ equals

$$
\begin{aligned}
T W_{3}\left(T^{*}\right)= & T W_{3}(G)+(p+q) D(w)+r\left(\sum_{i=1}^{p} a_{i}+\sum_{j=1}^{q} b_{j}\right) \\
& +\sum_{i<j}\left(a_{j}-a_{i}\right)+\sum_{i<j}\left(b_{j}-b_{i}\right)+q \cdot \sum_{i=1}^{p} a_{i}+p \cdot \sum_{j=1}^{q} b_{j},
\end{aligned}
$$


where $r \geq 1$ is the number of vertices of degree 3 in $G$.

After reattaching the caterpillar $C_{2}$ to the end of caterpillar $C_{1}$, the degree of vertex $w$ remains the same. The generalized terminal Wiener index of transformed tree $T^{\prime}$ equals

$$
\begin{aligned}
T W_{3}\left(T^{\prime}\right)= & T W_{3}(G)+(p+q) D(w)+r \cdot \sum_{i=1}^{p} a_{i}+r\left(q \cdot a_{p}+\sum_{j=1}^{q} b_{j}\right) \\
& +\sum_{i<j}\left(a_{j}-a_{i}\right)+\sum_{i<j}\left(b_{j}-b_{i}\right)+q \cdot\left(p \cdot a_{p}-\sum_{i=1}^{p} a_{i}\right)+p \cdot \sum_{j=1}^{q} b_{j}
\end{aligned}
$$

By subtraction, we get

$$
T W_{3}\left(T^{\prime}\right)-T W_{3}\left(T^{*}\right)=r q \cdot a_{p}+p q \cdot a_{p}-2 q \cdot \sum_{i=1}^{p} a_{i},
$$

Since $a_{p} \geq a_{i}$ for $i=1,2, \ldots, p$, we have

$$
\begin{aligned}
T W_{3}\left(T^{\prime}\right)-T W_{3}\left(T^{*}\right) & =q\left(a_{p}(r+p)-2 \sum_{i=1}^{p} a_{i}\right) \\
& \geq 2 q \cdot \sum_{i=1}^{p}\left(a_{p}-a_{i}\right) \geq 0
\end{aligned}
$$

with equality if and only if $p+r=2$. Since $p \geq q \geq 1$ and $r \geq 1$, the equality holds iff $p=q=r=1$.

It follows that using this transformation we can not decrease the generalized terminal Wiener index $T W_{3}$. If $\operatorname{deg}(w)>3$, we can remove the pendent edge and subdivide some edge $e$ such that both components of $T^{*}-e$ contain vertices of degree 3 , and further increase the generalized Wiener index. Therefore after these transformations, there is only one 3-bounded caterpillar under $w$.

Finally, we conclude that the extremal tree is a 3-bounded caterpillar, and it can be easily seen that the maximal value of $T W_{3}$ is achieved for caterpillar of the form $C_{n, k, p}$.

For $k=3$, from (11) we have

$$
f(p)= \begin{cases}\frac{1}{12} p\left(3 n p-4 p^{2}-2-6 p\right), & \text { if } p \text { is even } \\ \frac{1}{12}(p+1)(p-1)(3 n-4 p-6), & \text { if } p \text { is odd }\end{cases}
$$

and

$$
f(p)-f(p-2)= \begin{cases}(p-1)(n-2 p)-1, & \text { if } p \text { is even } \\ (p-1)(n-2 p), & \text { if } p \text { is odd, }\end{cases}
$$

which is greater than zero since $n \geq 2 p+2$. By direct verification, we get $f\left(\left\lfloor\frac{n}{2}\right\rfloor-1\right)>$ $f\left(\left\lfloor\frac{n}{2}\right\rfloor-2\right)$ for $n>4$.

Therefore, it holds

$$
T W_{3}(T) \leq T W_{3}\left(C_{n, 3,\lfloor n / 2\rfloor-1}\right)
$$

with equality if and only if $T \cong C_{n, 3,\lfloor n / 2\rfloor-1}$.

This can be further generalized - for all $k>3$ the caterpillar that maximizes the function $f(p)=T W_{k}\left(C_{n, k, p}\right)$ has maximal generalized terminal Wiener index among trees on $n$ vertices. 


\section{$5 \quad$ Calculating $T W_{k}$ of partial cubes}

The $n$-cube $Q_{n}$ is the graph whose vertex set consists of all binary $n$-tuples (hence $\left|V\left(Q_{n}\right)\right|=$ $2^{n}$ ), two vertices are adjacent if the corresponding tuples differ in precisely one position. The central metric feature of the $n$ cube is the fact that the distance between two vertices is equal to the number of positions in which they differ. A subgraph $H$ of a graph $G$ is called isometric if for any vertices $u$ and $v$ of $H, d_{H}(u, v)=d_{G}(u, v)$. Partial cubes are isometric subgraphs of hypercubes. Important examples of partial cubes are hypercubes, even cycles, (chemical) trees, median graphs, benzenoid systems, phenylenes. The Cartesian product of partial cubes is again a partial cubes.

Let $G$ be a connected graph. Then $e=x y$ and $f=u v$ are in the Djoković-Winkler $\Theta$ relation [25] if

$$
d(x, u)+d(y, v) \neq d(x, v)+d(y, u) .
$$

The relation $\Theta$ is always reflexive and symmetric, and is transitive on partial cubes. Therefore, $\Theta$ partitions the edge set of a partial cube $G$ into equivalence classes $F_{1}, F_{2}, \ldots, F_{s}$, called $\Theta$-classes (or cuts). For any $1 \leq i \leq s$, the graph $G-F_{i}$ consists of two connected components. The vertex sets of these components will be denoted with $W_{(i, 0)}$ and $W_{(i, 1)}$, because they can be described as the vertices whose $i$-th coordinate is 0 and 1 , respectively. The sets $W_{(i, \chi)}, 1 \leq i \leq s, \chi \in\{0,1\}$ are called halfspaces of $G$, while $W_{(i, 0)}$ and $W_{(i, 1)}$ are complementary halfspaces [17] and it holds $\left|W_{(i, 0)}\right|+\left|W_{(i, 1)}\right|=n$.

Let $G$ be a partial cube with halfspaces $W_{(i, \chi)}, 1 \leq i \leq s, \chi \in\{0,1\}$. For any $1 \leq i \leq s$ and any $\chi \in\{0,1\},\left|W_{(i, \chi)}\right|^{(k)}$ denotes the number of vertices with degree $k$ in $W_{(i, \chi)}$.

Theorem 5.1 Let $G$ be a partial cube with halfspaces $W_{(i, \chi)}, 1 \leq i \leq s, \chi \in\{0,1\}$. Then

$$
T W_{k}(G)=\sum_{i=1}^{s}\left|W_{(i, 0)}\right|^{(k)} \cdot\left|W_{(i, 1)}\right|^{(k)} .
$$

Proof. $G$ is a partial cube, hence vertices of $G$ can be considered as a binary $s$-tuple $u=$ $u_{1} u_{2} \ldots u_{s}$. Moreover, since $G$ is isometric in $Q_{s}$, the distance between two vertices is the number of positions in which they differ. Set $\delta(x, y)=0$ if $x=y$, and $\delta(x, y)=1$ for $x \neq y$. Then

$$
\begin{aligned}
T W_{k}(G) & =\sum_{\substack{u, v \in V(G) \\
\operatorname{deg}(u)=\operatorname{deg}(v)=k}} d(u, v) \\
& =\sum_{\begin{array}{c}
u, v \in V(G) \\
\operatorname{deg}(u)=\operatorname{deg}(v)=k
\end{array}} \sum_{i=1}^{s} \delta\left(u_{i}, v_{i}\right) \\
& =\sum_{i=1}^{s}\left(\sum_{\substack{u, v \in V(G) \\
\operatorname{deg}(u)=\operatorname{deg}(v)=k}} \delta\left(u_{i}, v_{i}\right)\right) \\
& =\sum_{i=1}^{s}\left|W_{(i, 0)}\right|^{(k)} \cdot\left|W_{(i, 1)}\right|^{(k)} .
\end{aligned}
$$

This completes the proof. 
An advantage of Theorem 5.1 comparing to computing $T W_{k}(G)$ by the definition is that we do not need to compute distances, but only to count vertices in the classes. This theorem can be considered as another instance of Klavžar "cut method". For its general description and an overview of its applications in chemical graph theory see survey [18].

As an example, we obtain a closed expression for $T W_{3}$ of the coronene/circumcoronene homologous series $H_{k}$. In Figure 4, $2 k-1$ horizontal elementary cuts of $H_{k}$ are presented. There exist two additional groups of $2 k-1$ equivalent cuts, obtained by rotating the former group by $\frac{\pi}{3}$ and $-\frac{\pi}{3}$. The number of vertices of $H_{k}$ equals $n_{k}=6 k^{2}$, while there are exactly $6 k$ vertices of degree two.

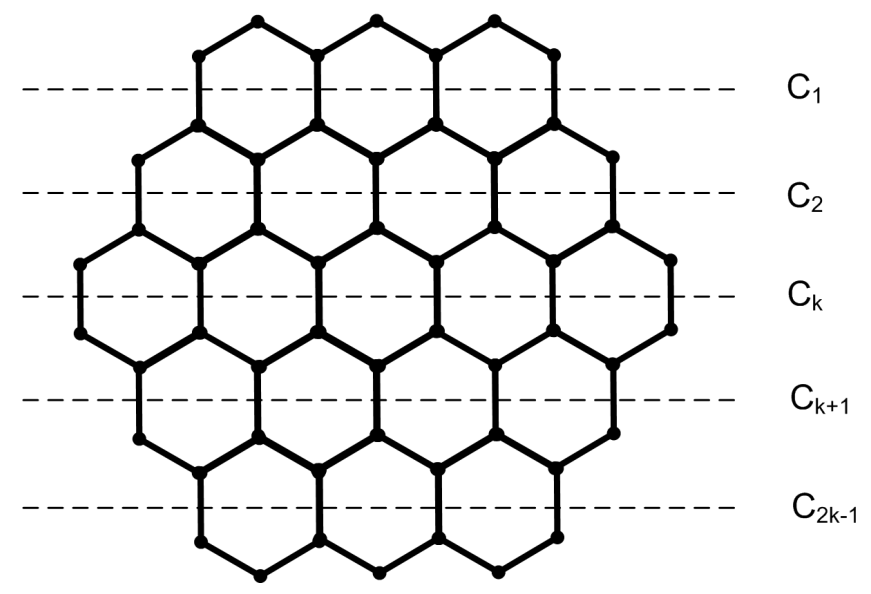

Figure 4: The Coronene / Circumcoronene $\mathrm{H}_{3}$.

Using symmetry, the contribution of the elementary cut $C_{i}$ is equal to the contribution of $C_{2 k-i}, i=1,2, \ldots, k-1$. By induction it follows that for $i=1,2, \ldots, k$ the number of vertices above cut $C_{i}$ equals $i(2 k+i)$, while the number of vertices of degree 2 equals $k+2 i$. Therefore by using Theorem 5.1 and described cuts, we have

$$
\begin{aligned}
\frac{1}{3} T W_{3}\left(H_{k}\right) & =\left(3 k^{2}-3 k\right)^{2}+2 \sum_{i=1}^{k-1}\left(2 k i+i^{2}-k-2 i\right)\left(6 k^{2}-6 k-2 k i-i^{2}+k+2 i\right) \\
& =\frac{164 k^{5}}{15}-\frac{82 k^{4}}{3}+\frac{58 k^{3}}{3}-\frac{5 k^{2}}{3}-\frac{19 k}{15} .
\end{aligned}
$$

Finally, we derive the fifth-order polynomial formula for the generalized terminal Wiener index of $H_{k}$

$$
T W_{3}\left(H_{k}\right)=\frac{1}{5}(k-1) k(2 k-1)\left(82 k^{2}-82 k-19\right) .
$$

Using similar methods as in [1, 16, 19] one can obtain the closed formulas for other chemical graphs (trees, benzenoid chains, phenylenes, ...) and design a linear algorithm for $T W_{k}$ of benzenoid systems.

\section{Concluding remarks}

The Wiener polarity index and the terminal Wiener index are very new molecular-structure descriptors and only a limited number of mathematical and chemical properties were established so far. In this paper we generalized these indices and open new perspectives for the future research. 
Another generalization of these indices may be the following

$$
W_{k}^{*}(G)=|\{(u, v) \mid d(u, v) \leq k, u, v \in V\}|=W_{1}(G)+W_{2}(G)+\ldots+W_{k}(G)
$$

and

$$
T W_{k}^{*}(G)=\sum_{\substack{u, v \in V(G) \\ \operatorname{deg}(u) \leq k, \operatorname{deg}(v) \leq k}} d(u, v) \geq T W_{1}(G)+T W_{2}(G)+\ldots+T W_{k}(G) .
$$

It would be nice to study mathematical and algorithmic properties of these indices and report their chemical relevance. These indices are obtained from the famous Wiener index, which has many applications in chemistry, graph theory and computer science.

Acknowledgement. This work was supported by Research Grants 174010 and 174033 of Serbian Ministry of Science. The authors are grateful to Nikola Milosavljević, Emeric Deutsch and Ivan Gutman for several useful suggestions while preparing the article.

\section{References}

[1] V. Chepoi, S. Klavžar, The Wiener index and the Szeged index of benzenoid systems in linear time, J. Chem. Inf. Comput. Sci. 37 (1997) 752-755.

[2] T. H. Cormen, C. E. Leiserson, R. L. Rivest, C. Stein, Introduction to Algorithms, Second Edition, MIT Press, 2001.

[3] P. Dankelmann, I. Gutman, S. Mukwembi, H. C. Swart, The edge-Wiener index of a graph, Discrete Math. 309 (2009) 3452-3457.

[4] H. Deng, On the extremal Wiener polarity index of chemical trees, MATCH Commun. Math. Comput. Chem. 60 (2011) 305-314.

[5] H. Deng, H. Xiao, F. Tang, The maximum Wiener polarity index of trees with $k$ pendants, Appl. Math. Letters 23 (2010) 710-715.

[6] H. Deng, H. Xiao, F. Tang, On the extremal Wiener polarity index of trees with a given diameter, MATCH Commun. Math. Comput. Chem. 63 (2010) 257-264.

[7] X. Deng, J. Zhang, Equiseparability on terminal Wiener index, in: A. V. Goldberg, Y. Zhou (Eds.), Algorithmic Aspects in Information and Management, Springer-Verlag, Berlin, 2009, 166-174.

[8] W. Du, X. Li, Y. Shi, Algorithms and extremal problem on Wiener polarity index, MATCH Commun. Math. Comput. Chem. 62 (2009) 235-244.

[9] A. A. Dobrynin, R. C. Entringer, I. Gutman, Wiener index of trees: theory and applications, Acta Appl. Math. 66 (2001) 211-249.

[10] I. Gutman, B. Furtula, M. Petrović, Terminal Wiener index, J. Math. Chem. 46 (2009) $522-531$.

[11] I. Gutman, O. E. Polansky, Mathematical Concepts in Organic Chemistry, Springer Verlag, Berlin, 1988. 
[12] H. Hosoya, Mathematical and chemical analysis of Wiener's polarity number, in: D. H. Rouvray, R. B. King (Eds.), Topology in Chemistry - Discrete Mathematics of Molecules, Horwood, Chichester, 2002.

[13] H. Hosoya, On some counting polynomials in chemistry, Discrete Appl. Math. 19 (1988) 239-257.

[14] A. Ilić, D. Stevanović, On Comparing Zagreb Indices, MATCH Commun. Math. Comput. Chem. 62 (2009) 681-687.

[15] A. Ilić, A. Ilić, D. Stevanović, On the Wiener index and Laplacian coefficients of graphs with given diameter or radius, MATCH Commun. Math. Comput. Chem. 63 (2010) 91-100.

[16] A. Ilić, S. Klavžar, D. Stevanović, Calculating the Degree Distance of Partial Hamming Graphs, MATCH Commun. Math. Comput. Chem. 63 (2010) 411-424.

[17] W. Imrich, S. Klavžar, Product Graphs: Structure and Recognition, John Wiley \& Sons, New York, 2000.

[18] S. Klavžar, Bird's eye view of the cut method and a survey of its applications in chemical graph theory, MATCH Commun. Math. Comput. Chem. 60 (2008) 255-274.

[19] S. Klavžar, I. Gutman, Wiener number of vertex-weighted graphs and a chemical application, Discrete Appl. Math. 80 (1997) 73-81.

[20] B. Liu, H. Hou, Y. Huang, On the Wiener polarity index of trees with maximum degree or given number of leaves, Comp. Math. Appl. 60 (2010) 2053-2057.

[21] S. Nikolić, G. Kovačević, A. Milićević, N. Trinajstić, The Zagreb indices 30 years after, Croat. Chem. Acta 76 (2003) 113-124.

[22] B. E. Sagan, Y. N. Yeh, P. Zhang, The Wiener polynomial of a graph, Int. J. Quantum Chem. 60 (1996) 959-969.

[23] L. A. Székely, H. Wang, T. Wu, The sum of the distances between the leaves of a tree and the 'semi-regular' property, Discrete Math. (2010), doi:10.1016/j.disc.2010.06.005.

[24] R. Todeschini, V. Consonni, Handbook of Molecular Descriptors, Wiley - VCH, Weinheim, 2000.

[25] P. Winkler, Isometric embeddings in products of complete graphs, Discrete Appl. Math. 7 (1984) 221-225.

[26] K. A. Zaretskii, Construction of trees using the distances between pendent vertices, Uspekhi Math. Nauk. 20 (1965) 90-92. 